\title{
Does pomegranate protect against gamma-rays-induced cardiac illnesses in rats?
}

\author{
Sameh Soliman Tawfik*, Ahmed Amer Elkady and Heba Hosny Mansour \\ Health Radiation Research Department, National Centre for Radiation Research and Technology (NCRRT), P.O.Box: 29, Nasr City, Egypt, Egyptian Atomic \\ Energy Authority, Cairo, Egypt
}

\begin{abstract}
Pomegranate is a heart healthy fruit juice. The study aims to evaluate the effectiveness of pomegranate powder extract (Pom) in preventing $\gamma$-rays-induced cardiac illnesses in rat model. Rats were randomly divided into four groups of 10 . Control group received $0.2 \mathrm{ml}$ diluted ethanol (vehicle) for 20days; Irradiated (IR) group received the vehicle for 10 days, exposed to $8 \mathrm{~Gy}-\gamma$-rays and then given the vehicle for additional 10days; Pom group received $50 \mathrm{mg}$ Pom $/ \mathrm{kg}$ body weight for $20 \mathrm{days}$, and Pom+IR group received Pom for 10days then, rats were exposed to 8Gy- $\gamma$-rays and received Pom for additional 10days. Irradiated rats showed increased lipid peroxidation (LPO) and total nitrate/nitrite $(\mathrm{NO}(\mathrm{x}))$, and decreased levels of reduced glutathione $(\mathrm{GSH})$ content, and the relative antioxidant enzyme activity of superoxide dismutase (SOD), glutathione peroxidase (GSHPx) and total antioxidant capacity (TAC) in the heart homogenates. Besides, asymmetric dimethylarginines (ADMA) was augmented in serum. Myocardial necrosis, edema, and inflammation were evident through the light microscope examination. Pre-treated rats with Pom significantly prevented or resisted all these alterations. Oral pre-treatment with Pom was effective in cardio protection in the $\gamma$-irradiated rats.
\end{abstract}

\section{Introduction}

Cardiovascular disease (CVD) is estimated to be the leading cause of death and loss of ability-adjusted life-years worldwide [1,2]. Recently, the clinical importance of radiation-induced heart diseases, particularly in postoperative mediastinal radiation therapy, has been recognized [3,4], these CVD complications include congestive heart failure, constrictive pericarditis, cardiomyopathy, coronary vascular disease, valvular heart disease, and sudden death [5].

Oxidative stress is well known to be involved in the pathophysiology of several disorders. Reactive oxygen species (ROS) are created naturally within cells to support biochemical activities, however when ROS are abundant, cellular antioxidant defense mechanisms are overcome, ending in a cascade of molecular damage [6,7]. Since ROS are the major mediators for radiation-induced damages [8]. A treatment combining radiation with an antioxidant might provide a strategy for preventing radiation injury to normal tissues [9].

In the Middle East and India, Pomegranate; Punica granatum L, a primaeval fruit, has constructive nutrients, many bioactive polyphenolic components, and essential phytochemicals. The studies have demonstrated that Pom improves blood lipid profiles and has antiinflammatory, antioxidant, anti-proliferative, anti-angiogenic, and antimetastatic properties [10]. These activate explain how Pom adds in the management against numerous adverse health effects, therefore, added the Pom fruit in person's regimen would afford a healthy life and would act as an effective therapy with no toxic side effects $[11,12]$.

Safety, low side effects, and the popularity of natural products have made them interesting for the researchers as an alternative source of curative agents [13]. The present study was designed to investigate the potential preventive effects of Pom against the $\gamma$-rays-induced cardiac illnesses in rats.

\section{Materials and methods}

\section{Animals}

Forty male Sprague-Dawley rats (age 10-11 weeks), weighing 280$300 \mathrm{~g}$, obtained from the Egyptian organization for biological product and vaccines, Giza, Egypt. Rats were preserved in normal conditions and were permitted free access to a normal diet and water ad libitum and kept under typical circumstances of humidity, temperature (20$24^{\circ} \mathrm{C}$ ), and 12-h light-dark cycle. Animals were deprived of food, but not water, overnight before investigations. All experiments were performed following Research Ethics Committee (REC-NCRRT) Number: $14 \mathrm{~A} / 18$, valid from 7/6/2018 at National Centre for Radiation Research and Technology (NCRRT) and adherence to the Principles of Laboratory Animal Care (NIH publication \#85-23, revised in 1985).

\section{Chemicals}

Pom powder was obtained from Natural in Bio-Resources Co., Ltd. (Changsha City, China). All other chemicals and solvents used were of the highest purity grade available.

\section{Radiation facility}

Irradiation was achieved with a Canadian gamma cell-40, $\left({ }^{137} \mathrm{Cs}\right)$ at the NCRRT, Nasr City, Cairo, Egypt. Animals were exposed to a single dose level of $8 \mathrm{~Gy} \gamma$-rays at a dose rate of $0.42 \mathrm{~Gy} / \mathrm{min}$ at the time of experimentation. There was anesthetization pre-gamma-rays exposure.

${ }^{*}$ Correspondence to: Sameh Soliman Tawfik, Health Radiation Research Department, National Centre for Radiation Research and Technology (NCRRT), P.O.Box: 29, Nasr City, Egypt, Egyptian Atomic Energy Authority, Cairo, Egypt, Email: samosoliman@gmail.com

Key words: pomegranate, radio-protector, cardiac-illnesses, $\gamma$-rays, rats

Received: May 26, 2020; Accepted: June 04, 2020; Published: June 10, 2020 


\section{Animal groups and experimental procedures}

Rats were haphazardly categorized into four groups of 10 . First, the control group, rats received $0.2 \mathrm{ml}$ diluted ethanol ( $1 \%$ ethanol in saline as a vehicle) for 20days. Second, irradiated (IR) group, rats received the vehicle for 10days and then exposed to whole-body $8 \mathrm{~Gy} \gamma$-rays and vehicle was maintained for 10additional days. Third, Pom group, rats received gavage of Pom (50mg/ kg body weight/day) for 20 days as Toklu et al. [14] recommended and the last group is Pom+IR, animals received Pom for 10days and one hour post the last Pom dosage, rats were exposed to $8 \mathrm{~Gy} \gamma$-rays then Pom was continued for additional 10 days. Rats were killed by cervical dislocation 24 hours post end of the trial after an overnight fast.

\section{Biochemical analysis}

Blood and heart tissue samples were collected from rats of different groups under standard laboratory conditions. The cardiac tissues were homogenate to assessment malondialdehyde (MDA) and reduced glutathione (GSH) levels, and the relative antioxidant enzyme activity of superoxide dismutase (SOD), glutathione peroxidase (GSHPx) and total antioxidant capacity (TAC) using commercial kits (Zellbio $\mathrm{GmbH}$, Germany) according to the manufacturer's instructions. The absorbance was read at $420 \mathrm{~m}, 412 \mathrm{~nm}, 535 \mathrm{~nm}, 412 \mathrm{~nm}$ and $490 \mathrm{~nm}$, respectively. Detection of total nitrate/nitrite $(\mathrm{NO}(\mathrm{x}))$ in the heart was performed by colorimetric technique (Oxford Biomedical Research, UK) according to the manufacturer's advice. The absorbance was read at $450 \mathrm{~m}$. Asymmetric dimethylarginines (ADMA), EpiCypher Inc, USA was detected according to the manufacturer's instructions.

\section{Histopathological finding}

The heart tissues were fixed in $10 \%$ neutral formalin reagent. After 72hours, tissues were dehydrated through a series of graded alcohol, embedded in paraffin, and cut into 4-micron sections and stained with hematoxylin and eosin (H\&E) according to Gamble [15] and examined microscopically.

\section{Statistical study}

The results obtained were expressed as Mean \pm S. D. The data were analyzed using one-way analysis of variance; ANOVA and post hoc multiple evaluations were completed with LSD test using SPSS/ PC software package (version 21; SPSS Inc., Chicago, IL, USA). The variance was considered significant at $P$-value $<0.05$.

\section{Results}

\section{Result of biochemical analysis}

As existing in Figures 1-3, the animal group treated with Pom only showed non-significant changes compared to the corresponding values of the control group in all estimated parameters in both serum and cardiac tissues of the trail. In the tissue of heart, the MDA content was markedly augmented, but GSH content was suggestively reduced in IR group as compared to the parallel rate of the control group. Pom+IR rat group established a significant decline in MDA level and a significant upgrade in GSH level as compared to the IR group, (Figures lA and 1B).

As shown in Figures $1 \mathrm{C}$ and 1D, the cardiac homogenates; SOD and GSHPx activities were significantly reduced in the IR group

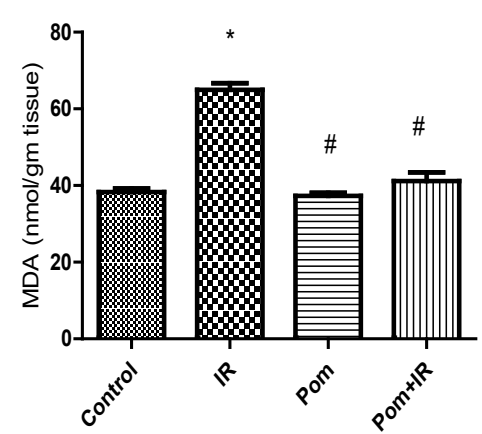

[A]

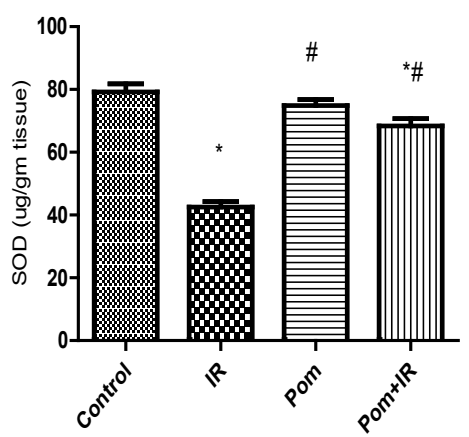

$[\mathrm{C}]$

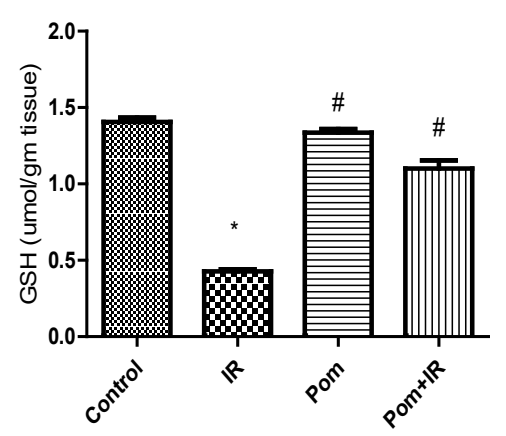

[B]

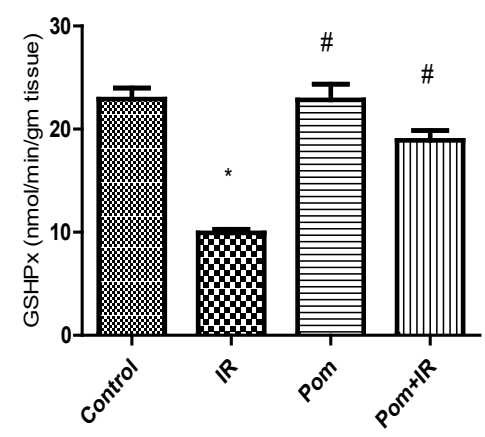

[D]

Figure 1. Effect of Pom (50mg/ kg body weight), IR (8Gy $\gamma$-rays), and their combination (Pom+IR) on [A] malondialdehyde (MDA) content, [B] reduced glutathione (GSH) content, [C] superoxide dismutase (SOD) activity and [D] glutathione peroxidase (GSHPx) activity in cardiac tissues

Data are presented as mean $\pm \mathrm{SD}, \mathrm{n}=10$. ** Indicate significant change from the both control group and IR group, respectively at $p \leq 0.05$ using one way ANOVA followed by Tukey-Kramer as a post-test 
as compared to the equivalent level in the control group. Significant increases in both of them were noticed in the Pom+IR group when compared to the IR group.

In the cardiac tissues, $\mathrm{NO}(\mathrm{x})$ concentration was significantly augmented, but TAC activity was significantly reduced in the IR group as compared to the matching rate of the control group (Figures $2 \mathrm{~A}$ and 2B). Pom + IR group established a significant decrease in $\mathrm{NO}(\mathrm{x})$ level and a significant increase in TAC activity as compared to the IR group (Figures $2 \mathrm{~A}$ and $2 \mathrm{~B}$ ).

In blood serum, the ADMA activity was significantly increased in the IR group as compared to the corresponding value of the control group (Figure 3). Pom+IR group established a significant decrease in ADMA activity as compared to the IR group (Figure 3).

\section{Histopathological results}

Light micrograph of myocytes in both control and Pom groups showed normal muscles with one or two large oval nuclei present at the central position. The perinuclear sarcoplasmic region is clear. The intercalated disks are asymmetrical and wider than normal crossstriations and represent specialized junctions between myocytes (Figure 4A). Pom group showed intact myocardial architecture (Figure 4B).
The IR group showed the myocardial muscles were necrotically changed and substituted by leukocytic cells in some cases (Figure 4C). Other cases showed dilated coronary vessels and hyalinization of cardiac muscles (Figure 4D). Furthermore, edema and hemorrhage of myocardial muscled were observed (Figure 4E). In most cases of the histological structure of the myocardial muscles in the Pom+IR group, showed relatively well-preserved architecture without necrosis, degenerative changes or hyalinization (Figure $4 \mathrm{~F}$ ). In a few cases, the preserved cardiac muscles were observed with mild edema and slight hemorrhage (Figure 4G).

\section{Discussion}

As heart illnesses were recently recognized in radiotherapeutic patients [16], antioxidant pre-treatment may attenuate cardiac dysfunction, cardiomyocytes damage and necrosis as well as other functional disturbances consequent [17]. Many manufactured drugs are used for the control of heart illnesses. However, they cannot meet the demands due to multiple etiological factors of these illnesses, and their certain adverse effects $[18,19]$. Therefore, we postulated that pre-treatment with polyphenolic antioxidants is a possible beneficial approach to restrict pathological changes and protect the heart from irradiation toxicity. Supplementary therapies using phytonutrients

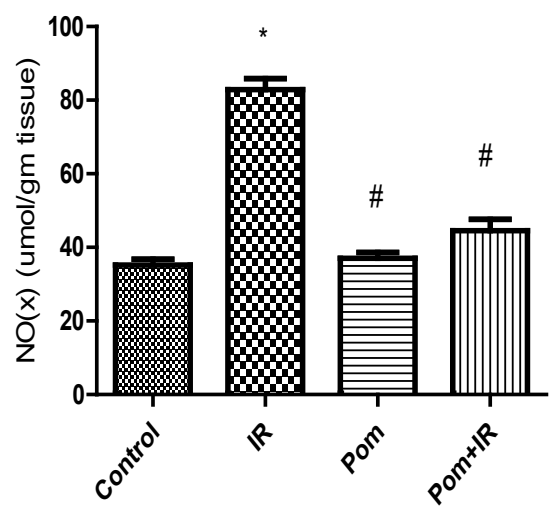

[A]

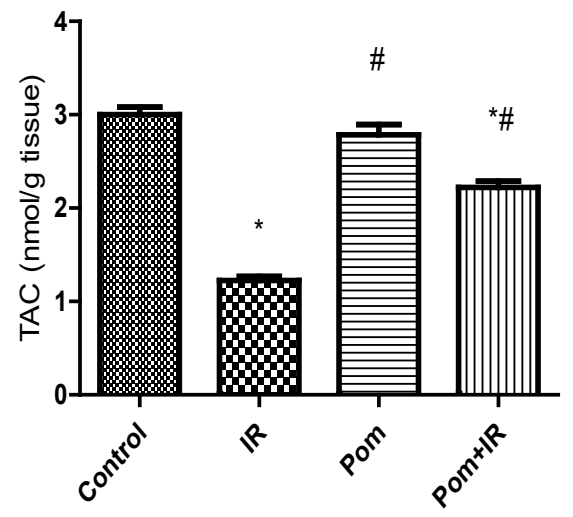

[B]

Figure 2. Effect of Pom (50mg/ kg body weight), IR (8Gy $\gamma$-rays), and their combination (Pom+IR) on [A] total nitrate/nitrite (NOx) content and [B] total antioxidant capacity (TAC) activity in cardiac tissues

Data are presented as mean $\pm \mathrm{SD}, \mathrm{n}=10$. *, Indicate significant change from the both control group and IR group, respectively at $p \leq 0.05$ using one way ANOVA followed by Tukey-Kramer as a post-test

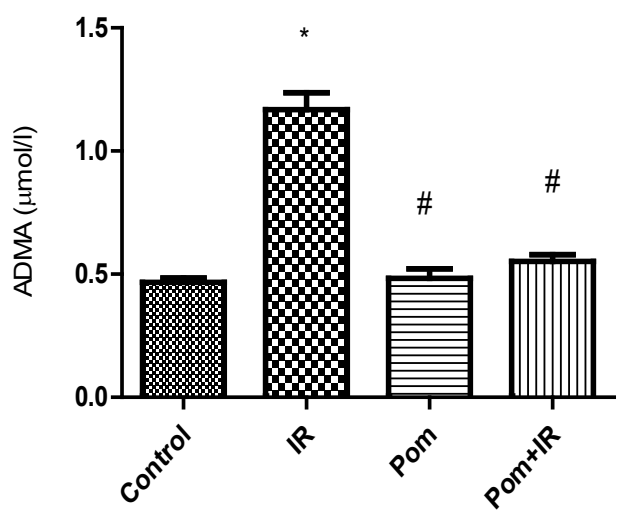

Figure 3. Effect of Pom (50mg/ kg body weight), IR (8Gy $\gamma$-rays), and their combination (Pom+IR) on asymmetric dimethylarginine (ADMA) activity in blood serum Data are presented as mean $\pm \mathrm{SD}, \mathrm{n}=10$. *,*Indicate significant change from the both control group and IR group, respectively at $p \leq 0.05$ using one way ANOVA followed by Tukey-Kramer as a post-test 


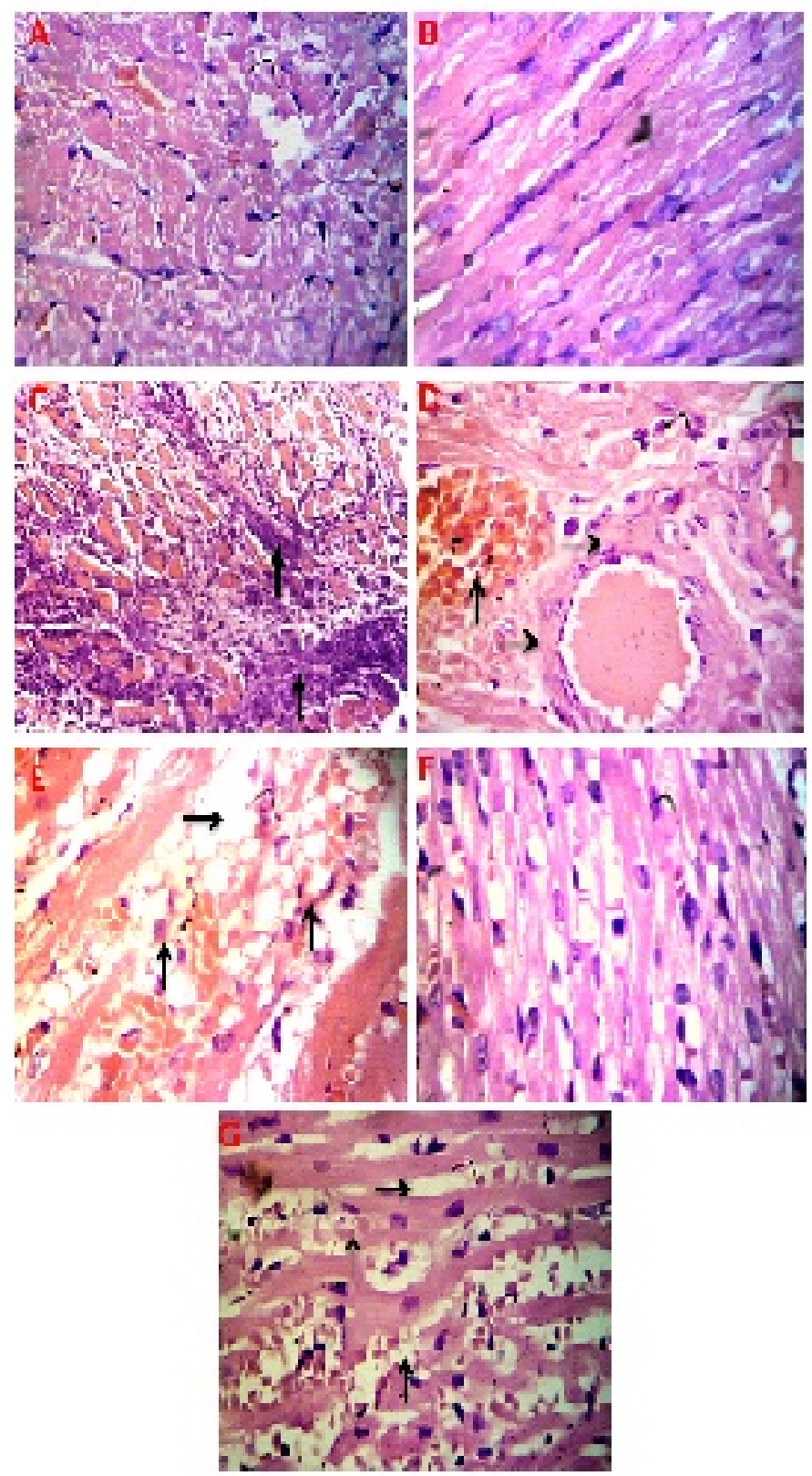

Figure 4. (A) Heart of the control group: Rat showing the normal structure (B) Heart of the Pom group: Rat showing the normal structure (C) Heart of the IR group: Rat showing necrotic myocardial muscle fiber covered with leukocytic infiltration $(\Lambda)(D)$ Heart of the IR group: Rat showing ongested blood vessel $(\uparrow)$ and hyalinized myocardial muscle fiber $(\rightarrow)($ E) Heart of the IR group: Rats showing edema $(\rightarrow)$ and elimination of necrotic myocytes by phagocytosis ( $\uparrow$ ) (F) Heart of the Pom+IR group: Rat showing normal myocytes and $(\mathrm{G})$ Heart of the Pom+IR group: Rat showing mild edema $(\rightarrow)$ and slight hemorrhage ( $\uparrow),(\mathrm{H} \& \mathrm{E} \times 400)$

are applied progressively as these nutrients have no or minor side effects and are cost dynamic. Besides, polyphenols are excellent cardio protectants $[20,21]$.

Pom is a highly valued plant, Ancient Egyptians regarded the pomegranate as a symbol of prosperity and ambition and the Jewish Torah is regarded to confer powers of fruitfulness [11]. The remedial uses of Pom have a long history; conferring to the Ebers Papyrus, one of the oldest medical works of literature from around $1500 \mathrm{BC}$, Egyptians used the Pom for curing of tapeworm and other infections
[22]. A current study indicated that Pom exerted protective effects against oxidative stresses and cellular damages in kidney, liver and heart induced by lead in rats [23]. Pom is a rich source of polyphenolic compound content and antioxidant such as ellagic, tannin, gallotannins and anthocyanins, and flavonoids. Also, Pom juice demonstrates a significantly higher antioxidant activity compared to green tea [24,25]. The present results demonstrate that Pom is cardioprotective in acute heart cellular and biochemical alterations induced by $\gamma$-rays in rats. This is manifested by improved cardiac function, reduced oxidative stress and improved antioxidant defense. Ismail et al. [26] found that ROS emitting from ionizing radiation exposure are well-documented in chronic diseases, including CVD and cancer.

Increased LPO is generally believed to be an important primary cause of the initiation of oxidative stress related to various tissue injuries, cell death, and further progression of many acute and chronic diseases [27]. The study indicated a rise in the level of cardiac MDA as an indication of LPO in IR-group, but pre-treatment with Pom significantly decreased its level. This result is in agreement with that of El-Mansi and Al-Kahtani [28], they discovered that Pom has a protective influence against cardiomyocytes' oxidative stress.

GSH acts as an antioxidant both intracellularly and extracellularly in concurrence with numerous enzymatic processes that reduce hydrogen peroxide and hydro-peroxides [29]. Gamma-rays-induced cardiac toxicity is associated with reduced antioxidant levels [30]. Exposed to $\gamma$-rays significantly lowered the GSH level. While, pre-treatment with Pom, improved the level of GSH in the heart. Aksu et al. [23], found that Pom has a protective role against the cellular injury induced by lead in the heart tissue of rats. Moreover, the increased in GSH level may be due to induction of GSH-biosynthesis enzymes that are known to be increased by plant polyphenol [31].

The important protective enzymes against ROS are SOD and GSHPx [32]. In the present study their activities were reduced due to $\gamma$-rays exposure in the IR group. These results were similar to that of other studies [33,34]. The two antioxidant enzymes were augmented in Pom+IR group. This augmentation may be due to increases in antioxidant enzymes activity in tissues by Pom [35].

A significant increase in TAC values after pre-treatment with Pom was observed in Pom+IR group compared with the IR group. The reduction in ROS after pre-treatment with Pom is especially caused by increases in antioxidant enzymes activity in cardiac tissue marker; TAC [36], furthermore, Hassan et al. [37] data revealed that ingestion of Pom resulted in a significant rise in plasma TAC of growing rabbits.

NO is a free radical that assists as a crucial messenger particle in inflammatory disorders. Decreased production of NO reduces the inflammatory symptoms and gives protection [38]. Moreover, NO plays multifaceted roles in combating cardiovascular diseases [39]. Intake of polyphenol-rich foodstuffs can moderate CVD hazards by collection NO and ease of its bioavailability [40]. We showed that Pom inhibited NO generation in cardiac tissue compared to IR-group. Pinheiro et al. [41] found that Punica granatum L. reduced NO production in acute lung injury and concluded that the prophylactic treatment with Pom attenuates acute lung inflammation in mice. In addition, Shirbazou and Jafari [42] postulated that Pom may also affect the regulation of pathological conditions caused by excessive generation of NO and its oxidation product peroxynitrite and suggested the Pom applicability as a potent and novel therapeutic agent for scavenging NO from lung tissue. A significant increase in NO production in heart tissue was showed under ischemic conditions [43]. Moreover, Hosny Mansour and 
Farouk Hafez [44] observations, suggested that gamma-rays improved endogenous NO-synthesis in liver, intestine, lung, kidney, brain, spleen or heart tissue of the animals by facilitating the entry of $\mathrm{Ca}^{2+}$ ions into the membrane and the cells produce NO.

ADMA is a marker of endothelial dysfunction and is measured for follow-up heart muscle stroke [45]. Moreover, ADMA is revealed as an independent early marker of CVD [46]. Also, it was reported elevated plasma ADMA level in cases of risk factors for cardiovascular diseases in the rat [47]. The results of this study indicated a significant increase in serum ADMA level in IR group and a significant reduction in its value in Pom+IR group. This decrease was obtained due to supplementing Pom as a pre-treatment for irradiated rats. Pom is a highly concentrated source of dietary polyphenols. Trexler et al. [48] indicating enhanced vasodilation and blood flow in response to nitrate and polyphenols. It was recommended that polyphenols increase intermediated vasodilation flow [49]. Additionally, enhancing the nourishment with polyphenol-rich bee pollen ethanol extract resulted in a reduction of ADMA level which evidences a protective role against hepatic steatosis and deteriorating changes caused by a high-fat regime in mice [50].

Histopathology of the myocardium of the IR group showed extensive myocytes necrosis, edema, and myofibril disorganization. This result is similar to cardiotoxicity induced by irradiation [51]. However, Pom pretreatment in $\gamma$-rays-challenged heart showed reduced myocytes necrosis and cardiac damage as well as myocardial recovery. Aksu et al. [23] suggested the protective effect of supplementation with Pom against lead acetate-induced cardiac, kidney and liver histopathological necrosis in rats. Moreover, Pom can confer cardioprotective effects [52]. In addition, Zhao et al. [53] indicated that polyphenols' rich fruits help in CVD regulation and also proved that Pom exerted its cardiovascular protective action by scavenging excess free radicals, reducing oxidative stresses and increasing the antioxidant defense system. Themyocardium of the group of rats treated with Pom showed normal cardiac fibres without any pathological changes. This indicates that Pom does not possess any adverse effects under normal conditions. Thus, the histopathological and biochemical observations of the present study strongly support and confirm the Pom properties.

\section{Conclusion}

Therefore, pre- and post-treatment with Pom significantly attenuates the cardiotoxic effects of $\gamma$-rays through significant reduction of cardiac injury biomarkers, lipid peroxidation, and rise antioxidant enzymes; this effect may relate to the polyphenolic compounds which have significant biological activity including antioxidant effect. Further researches are required to fully recognize the mode of action of the dynamic components and to entirely fulfilment pomegranate's protective, curative and beneficial potential.

\section{Acknowledgements}

We are thankful to our colleagues in Health Radiation Research Department for providing laboratory services, and technicians of the National Centre for Radiation Research and Technology (NCRRT), Egyptian Atomic Energy Authority, for providing the fundamental irradiation facilities. This work was done in NCRRT, Egypt.

\section{Conflict of interest announcement}

The authors state that their no conflicts of interest. The authors alone are responsible for the content and manuscript of the article.

\section{References}

1. Jódar E, Michelsen M, Polonsky WH, Réa R, Sandberg A (2020) Semaglutide improves health-related quality of life vs placebo when added to standard of care in patients with type 2 diabetes at high cardiovascular risk (SUSTAIN 6). Diabetes Obes Metab 22: 1339-1347. [Crossref]

2. Sørensen TB, Matsuzaki M, Gregson J, Kinra S, Kadiyala S (2020) Is agricultura engagement associated with lower incidence or prevalence of cardiovascular diseases and cardiovascular disease risk factors? A systematic review of observational studies from low- and middle-income countries. PLoS One 15: e0230744.

3. Huang S, Che J, Chu Q, Zhang P (2020) The Role of NLRP3 inflammasome in radiation-induced cardiovascular injury. Front Cell Dev Biol 8: 140.

4. Lee Chuy K, Nahhas O, Dominic P, Lopez C, Tonorezos E (2019) Cardiovascular complications associated with mediastinal radiation. Curr Treat Options Cardiovasc Med 21: 31 .

5. Aronson D, Brenner B (2018) Arterial thrombosis and cancer. Thromb Res 1: S23-S28

6. Guo SW (2020) Cancer-associated mutations in endometriosis: shedding light on the pathogenesis and pathophysiology. Hum Reprod Update 26: 423-449.

7. Sies H, Jones DP (2020) Reactive oxygen species (ROS) as pleiotropic physiological signalling agents. Nat Rev Mol Cell Biol 21: 363-383.

8. Khodamoradi E, Hoseini-Ghahfarokhi M, Amini P, Motevaseli E, Shabeeb D (2020) Targets for protection and mitigation of radiation injury. Cell Mol Life Sci 77: 3129 3159.

9. Farhood B, Aliasgharzadeh A, Amini P, Saffar H, Motevaseli E (2019) Radiationinduced dual oxidase upregulation in rat heart tissues: protective effect of melatonin. Medicina (Kaunas) 55: E317.

10. Baradaran Rahimi V, Ghadiri M, Ramezani M, Askari VR (2020) Anti-inflammatory and anti-cancer activities of pomegranate and its constituent, ellagic acid: Evidence from cellular, animal, and clinical studies. Phytother Res 34: 685-720.

11. Medjakovic S, Jungbauer A (2013) Pomegranate: a fruit that ameliorates metabolic syndrome. Food Funct 4: 19-39.

12. Soleimanian Y, Goli SAH, Varshosaz J, Maestrelli F (2018) Propolis wax nanostructured lipid carrier for delivery of $\beta$ sitosterol: Effect of formulation variables on physicochemical properties. Food Chem 260: 97-105.

13. Afshari AR, Sadeghnia HR, Mollazadeh H (2016) A review on potential mechanisms of terminalia chebula in Alzheimer's disease. Adv Pharmacol Sci 2016: 8964849.

14. Toklu HZ, Sehirli O, Ozyurt H, Mayadağli AA, Ekşioğlu-Demiralp E (2009) Punica granatum peel extract protects against ionizing radiation-induced enteritis and leukocyte apoptosis in rats. J Radiat Res 50: 345-353.

15. Gamble M (2008) Theory and practice of histological techniques. 6 ed. Philadelphia Churchill Livingstone/ Elsevier, p. 121-134.

16. Ping Z, Peng Y, Lang H, Xinyong C, Zhiyi Z (2020) Oxidative stress in radiationinduced cardiotoxicity. Oxid Med Cell Longev 2020: 3579143. [Crossref]

17. Boarescu PM, Boarescu I, Bocșan IC, Pop RM, Gheban D (2019) Curcumin nanoparticles protect against isoproterenol induced myocardial infarction by alleviating myocardial tissue oxidative stress, electrocardiogram, and biological changes. Molecules 24: E2802.

18. Guan S, Li J, Zhang K, Li J (2020) Environmentally responsive hydrogels for repair of cardiovascular tissue. Heart Fail Rev Feb 19.

19. Li L, Meng F, Zhu S, Guo S, Wang Y (2018) Efficacy and safety of WEI BI MEI, a chinese herb compound, as an alternative to bismuth for eradication of Helicobacter pylori. Evid Based Complement Alternat Med 2018: 4320219.

20. Sanches-Silva A, Testai L, Nabavi SF, Battino M, Pandima Devi K (2020) Therapeutic potential of polyphenols in cardiovascular diseases: Regulation of mTOR signaling pathway. Pharmacol Res 152: 104626.

21. Sun ZM, Guan P, Luo LF, Qin LY, Wang N (2020) Resveratrol protects against CIHinduced myocardial injury by targeting Nrf2 and blocking NLRP3 inflammasome activation. Life Sci 245: 117362

22. Jayaprakasha GKm Negi PS, Jena BS (2006) "Antimicrobial activities of pomegranate". In Seeram, Navindra P.; Schulman, Risa N.; Heber, David. Pomegranates: ancient roots to modern medicine. CRC/Taylor \& Francis Press. P. 168

23. Aksu DS, Sağlam YS, Yildirim S, Aksu T (2017) Effect of pomegranate (Punica granatum L.) juice on kidney, liver, heart and testis histopathological changes, and the tissues lipid peroxidation and antioxidant status in lead acetate-treated rats. Cell Mol Biol 63: 33-42. 
24. Bar-Ya'akov I, Tian L, Amir R, Holland D (2019) Primary metabolites, anthocyanins, and hydrolyzable tannins in the pomegranate fruit. Front Plant Sci 10: 620.

25. Rodriguez J, Gilson H, Jamart C, Naslain D, Pierre N (2015) Pomegranate and green tea extracts protect against ER stress induced by a high-fat diet in skeletal muscle of mice. Eur J Nutr 54: 377-389.

26. Ismail T, Sestili P, Akhtar S (2012) Pomegranate peel and fruit extracts: A review of potential anti-inflammatory and anti-infective effects. J Ethnopharmacol 143: 397-405.

27. Suman S, Kumar S, Fornace AJ Jr, Datta K (2018) The effect of carbon irradiation is associated with greater oxidative stress in mouse intestine and colon relative to $\gamma$-rays. Free Radic Res 52: 556-567.

28. El-Mansi AA, Al-Kahtani MA (2019) Calcitriol and Punica Granatum extract concomitantly attenuate cardiomyopathy of diabetic mother rats and their neonates via activation of Raf/MEK/ERK signalling and mitigation of apoptotic pathways. Folia Biol (Praha) 65: 70-87.

29. Sayed AA, Abbas OA, Saad MA, Marie MS (2018) Cicer arietinum extract ameliorate $\gamma$-irradiation disorders via modulation of oxidative/antioxidative pathway. $J$ Photochem Photobiol 183: 46-56.

30. Kura B, Kalocayova B, LeBaron TW, Frimmel K, Buday J (2019) Regulation of microRNAs by molecular hydrogen contributes to the prevention of radiation-induced damage in the rat myocardium. Mol Cell Biochem 457: 61-72.

31. Jeon Y, Kwak SS, Cheong SA, Seong YH, Hyun SH (2013) Effect of trans- $\varepsilon$-viniferin on in vitro porcine oocyte maturation and subsequent developmental competence in preimplantation embryos. J Vet Med Sci 75: 1277-1286.

32. Lin X, Bai D, Wei Z, Zhang Y, Huang Y (2019) Curcumin attenuates oxidative stress in RAW264.7 cells by increasing the activity of antioxidant enzymes and activating the Nrf2-Keap1 pathway. PLoS One 14: e216711.

33. Khedr MH, Shafaa MW, Abdel-Ghaffar A, Saleh A (2018) Radioprotective efficacy of Ginkgo biloba and Angelica archangelica extract against technetium-99m-sestamibi induced oxidative stress and lens injury in rats. Int J Radiat Biol 94: 37-44.

34. Tawfik SS, Elkady AA, El Khouly WA (2019) Crocin mitigates $\gamma$-rays-induced hepatic toxicity in rats. Environ Sci Pollut Res Int 26: 15414-15419.

35. Mollazadeh H, Boroushaki MT, Soukhtanloo M, Afshari AR, Vahedi MM (2017) Effects of pomegranate seed oil on oxidant/antioxidant balance in heart and kidney homogenates and mitochondria of diabetic rats and high glucose-treated $\mathrm{H} 9 \mathrm{c} 2$ cell line. Avicenna J Phytomed 7: 317-333.

36. Morvaridzadeh M, Sepidarkish M, Daneshzad E, Akbari A, Mobini GR (2020) The effect of pomegranate on oxidative stress parameters: A systematic review and metaanalysis. Complement Ther Med 48: 102252.

37. Hassan FA, Ibrahim MRM, Arafa SA (2020) Effect of dietary pomegranate by-product extract supplementation on growth performance, digestibility, and antioxidant status of growing rabbit. Trop Anim Health Prod 52: 1893-1901.

38. Bai L, Gao J, Wei F, Zhao J, Wang D (2018) Therapeutic potential of ginsenosides as an adjuvant treatment for diabetes. Front Pharmacol 9: 423.
39. Grandvuillemin I, Buffat C, Boubred F, Lamy E, Fromonot J (2018) Arginase upregulation and eNOS uncoupling contribute to impaired endothelium-dependent vasodilation in a rat model of intrauterine growth restriction. Am J Physiol Regul Integr Comp Physiol 315: R509-R520.

40. Taguchi C, Kishimoto Y, Fukushima Y, Kondo K, Yamakawa M (2020) Dietary intake of total polyphenols and the risk of all-cause and specific-cause mortality in Japanese adults: the Takayama study. Eur J Nutr 59: 1263-1271.

41. Pinheiro AJMCR, Gonçalves JS, Dourado ÁWA, de Sousa EM, Brito NM (2018) Punica granatum L. leaf extract attenuates lung inflammation in mice with acute lung injury. J Immunol Res 2018: 6879183.

42. Shirbazou Sh, Jafari M (2012) The multiple forms of Leishmania major in BALB/C mice lung in Iran. Iran J Parasitol 7: 99-102.

43. Ardjmand A, Shahaboddin ME, Mazoochi T, Ghavipanjeh G (2019) Ameliorative effects of cerebrolysin against isoproterenol-induced myocardial injury in male rats. Life Sci 227: 187-192.

44. Hosny Mansour H, Farouk Hafez H (2012) Protective effect of Withania somnifera against radiation-induced hepatotoxicity in rats. Ecotoxicol Environ Saf 80: 14-19.

45. Ziegler NL, Sieweke JT, Biber S, Gabriel MM, Schuppner R (2019) Markers of endothelial pathology to support detection of atrial fibrillation in embolic stroke of undetermined source. Sci Rep 9: 19424

46. Oliva-Damaso E, Oliva-Damaso N, Rodriguez-Esparragon F, Payan J, BaamondeLaborda E (2019) Asymmetric (ADMA) and Symmetric (SDMA) Dimethylarginines in chronic kidney disease: A clinical approach. Int J Mol Sci 20: E3668.

47. Wilcox CS (2012) Asymmetric dimethylarginine and reactive oxygen species: unwelcome twin visitors to the cardiovascular and kidney disease tables. Hypertension 59: $375-381$.

48. Trexler ET, Smith-Ryan AE, Melvin MN, Roelofs EJ, Wingfield HL (2014) Effects of pomegranate extract on blood flow and running time to exhaustion. Appl Physiol Nutr Metab 39: 1038-1042.

49. Barona J, Aristizabal JC, Blesso CN, Volek JS, Fernandez ML (2012) Grape polyphenols reduce blood pressure and increase flow-mediated vasodilation in men with metabolic syndrome. J Nutr 142: 1626-1632.

50. Rzepecka-Stojko A, Kabała-Dzik A, Kubina R, Jasik K, Kajor M (2018) Protective effect of polyphenol-rich extract from Bee Pollen in a High-fat diet. Molecules 23: E805.

51. Gurses I, Ozeren M, Serin M, Yucel N, Erkal HS (2018) Histopathological efficiency of amifostine in radiation-induced heart disease in rats. Bratisl Lek Listy 119: 54-59.

52. Thotambailu AM, Bhandary BSK, Sharmila KP (2019) Protective effect of punica granatum extract in head and neck cancer patients undergoing radiotherapy. Indian $J$ Otolaryngol Head Neck Surg 71: 318-320.

53. Zhao CN, Meng X, Li Y, Li S, Liu Q (2017) Fruits for prevention and treatment of cardiovascular diseases. Nutrients 9: E598.

Copyright: (C2020 Tawfik SS. This is an open-access article distributed under the terms of the Creative Commons Attribution License, which permits unrestricted use, distribution, and reproduction in any medium, provided the original author and source are credited. 